\title{
A LIOUVILLE-TYPE RESULT FOR NON-COOPERATIVE FISHER-KPP SYSTEMS AND NONLOCAL EQUATIONS IN CYLINDERS
}

\author{
LÉO GIRARDIN AND QUENTIN GRIETTE
}

\begin{abstract}
We address the uniqueness of the nonzero stationary state for a reaction-diffusion system of Fisher-KPP type that does not satisfy the comparison principle. Although the uniqueness is false in general, it turns out to be true under biologically natural assumptions on the parameters. This Liouville-type result is then used to characterize the wake of traveling waves. All results are extended to an analogous nonlocal reaction-diffusion equation that contains as a particular case the cane toads equation with bounded traits.
\end{abstract}

\section{INTRODUCTION}

We investigate the reaction-diffusion system

$$
\partial_{t} \mathbf{u}-\mathbf{D} \partial_{x x} \mathbf{u}=\mathbf{M u}+\mathbf{u}-\mathbf{u} \circ(\mathbf{C u}),
$$

where $t \in \mathbb{R}$ is a time variable, $x \in \mathbb{R}$ is a space variable, $\mathbf{u}(t, x)$ is a nonnegative column vector ${ }^{1}$ collecting $N \geq 2$ phenotype densities among a species, $\mathbf{D}$ is a diagonal matrix collecting positive diffusion rates, $\circ$ is the Hadamard product (componentby-component product) between two vectors and $\mathbf{M}$ and $\mathbf{C}$ are square matrices collecting respectively mutation rates and competition rates and satisfying the following standing assumptions (below and in the whole paper, $\mathbf{1}=(1,1, \ldots, 1)^{\mathrm{T}} \in \mathbb{R}^{N}$ ).

$\left(A_{1}\right)$ The matrix $\mathbf{M} \in \mathscr{M}_{N, N}(\mathbb{R})$ is essentially nonnegative (namely, with nonnegative off-diagonal coefficients), irreducible, line-sum-symmetric (namely, $\mathbf{M 1}=\mathbf{M}^{\mathrm{T}} \mathbf{1}$ ) and admits $(0, \mathbf{1})$ as Perron-Frobenius eigenpair (namely, M1 $=0)$.

$\left(A_{2}\right)$ The matrix $\mathbf{C} \in \mathscr{M}_{N, N}(\mathbb{R})$ is positive, normal and admits $(1, \mathbf{1})$ as PerronFrobenius eigenpair (namely, C1 $=\mathbf{1}$ ). We denote $\mathbf{U} \in \mathscr{M}_{N, N}(\mathbb{C})$ the unitary matrix such that $\mathbf{U} \mathbf{C} \mathbf{U}^{-1}=\mathbf{U} \mathbf{C} \overline{\mathbf{U}}^{\mathrm{T}}$ is diagonal.

$\left(A_{3}\right)$ The spectrum of $\mathbf{C}$ is contained in the complex closed right-half plane.

We are interested more specifically in the associated traveling wave equation

$$
-\mathbf{D} \mathbf{p}^{\prime \prime}-c \mathbf{p}^{\prime}=\mathbf{M p}+\mathbf{p}-\mathbf{p} \circ(\mathbf{C p})
$$

(L. G.) Université Paris-Saclay, CNRS, Laboratoire de Mathématiques D'Orsay, 91405 Orsay Cedex, France

(Q. G.) Université de Bordeaux, Institut de Mathématiques de Bordeaux, Bât. A33 Bureau 211, 351, cours de la Libération, 33405 Talence, France

E-mail addresses: leo.girardin@math.u-psud.fr, quentin.griette@math.u-bordeaux.fr.

2010 Mathematics Subject Classification. 35K40, 35K57, 92D25.

Key words and phrases. KPP nonlinearity, reaction-diffusion system, cane toads equation, Liouville-type result, traveling wave.

This work was supported by a public grant as part of the Investissement d'avenir project, reference ANR-11-LABX-0056-LMH, LabEx LMH. This work has been carried out in the framework of the NONLOCAL project (ANR-14-CE25-0013) funded by the French National Research Agency (ANR).

${ }^{1}$ In the whole paper, nonnegativity and positivity of vectors and matrices are understood component-wise. 
satisfied by solutions of the system (1) of the form $\mathbf{u}:(t, x) \mapsto \mathbf{p}(x-c t)$. This equation might be supplemented with asymptotic conditions for the profile $\mathbf{p}$. The asymptotic conditions of classical traveling waves $(\mathbf{p}, c)$ [25] are

$$
\lim _{+\infty} \mathbf{p}=\mathbf{0}, \quad \min _{i \in[N]} \liminf _{-\infty} p_{i} \geq 0, \quad \max _{i \in[N]} \liminf _{-\infty} p_{i}>0,
$$

where $[N]$ denotes (here and in the rest of the paper) the set $\{1,2, \ldots, N\}$.

By $\left(A_{1}\right)$ and $\left(A_{2}\right), 1$ is a constant steady state of the system (1).

1.1. Main results. Our main result is the following theorem.

Theorem 1.1 (Liouville-type result). Assume $\left(A_{1}\right),\left(A_{2}\right)$ and $\left(A_{3}\right)$, Then, for any $c \in \mathbb{R}, 1$ is the unique bounded solution $\mathbf{p}$ of (2) such that $\min _{i \in[N]} \inf _{\mathbb{R}} p_{i}>0$.

The main consequences of this theorem are the two following corollaries, deduced from standard elliptic estimates and limiting procedures 23] as well as a strong positivity property 25 , Theorem 1.1].

Corollary 1.2 (Uniqueness of the nonzero steady state). Assume $\left(A_{1}\right),\left(A_{2}\right)$ and $\left(A_{3}\right)$. Then 1 is the unique bounded nonnegative nonzero stationary solution of (1), namely the unique bounded nonnegative nonzero solution $\mathbf{p}$ of (2) with $c=0$.

Corollary 1.3 (Limit behavior of the traveling waves). Assume $\left(A_{1}\right),\left(A_{2}\right)$ and $\left(A_{3}\right)$. Then all bounded solutions $(\mathbf{p}, c)$ of (2)-(3) actually satisfy $\lim _{-\infty} \mathbf{p}=\mathbf{1}$.

1.2. Extension to nonlocal equations. Those results extend to continuous limits $N \rightarrow+\infty$, provided the limit equation has a similar structure. Below we illustrate this principle by focusing on an equation supplemented with Neumann boundary conditions, though it would also be possible to adapt our arguments in the periodic framework with no additional difficulty.

We consider

(4) $-d(y) \partial_{\xi \xi} p-c \partial_{\xi} p=\nabla_{y} \cdot\left(\sigma(y) \nabla_{y} p\right)+M[p(\xi)](y)+p(\xi, y)(1-K[p(\xi)](y))$

set on $(\xi, y) \in \mathbb{R} \times \Omega$ for a smooth domain $\Omega \subset \mathbb{R}^{Q}\left(Q \geq 1\right.$ and $\partial \Omega$ is $\left.\mathscr{C}^{2}\right)$ and supplemented with homogeneous Neumann boundary conditions at $y \in \partial \Omega$. Above, $d \in \mathscr{C}(\bar{\Omega},(0,+\infty)), \sigma \in \mathscr{C}^{1}(\bar{\Omega},(0,+\infty))$,

$$
M[p(\xi)]=\int_{\Omega} m(\cdot, \tilde{y})(p(\xi, \tilde{y})-p(\xi, \cdot)) \mathrm{d} \tilde{y}, \quad K[p(\xi)]=\int_{\Omega} k(\cdot, \tilde{y}) p(\xi, \tilde{y}) \mathrm{d} \tilde{y},
$$

for some $m, k \in \mathscr{C}\left(\Omega^{2},(0,+\infty)\right)$. Defining naturally the adjoint operators $M^{\star}$ and $K^{\star}$, the assumptions $\left.\left(A_{1}\right)\right]\left(A_{2}\right)$ and $\left(A_{3}\right)$ extend to the continuous equation as follows:

$\left(A_{1}^{\prime}\right)$ The function $\sigma(y) \in \mathscr{C}^{1}(\bar{\Omega})$ is positive and the function $m \in \mathscr{C}\left(\Omega^{2}\right)$ is nonnegative, bounded and satisfies $\int_{\Omega} m(\cdot, z) \mathrm{d} z=\int_{\Omega} m(z, \cdot) \mathrm{d} z$.

$\left(A_{2}^{\prime}\right)$ The function $k \in \mathscr{C}\left(\bar{\Omega}^{2}\right)$ is positive and the induced operator $K[p]=$ $\int_{\Omega} k(\cdot, z) p(z) \mathrm{d} z$ acting on the Hilbert space $L^{2}(\Omega)$ is normal. Moreover, the constant function $y \in \Omega \mapsto 1$ is an eigenvector of $K$ associated with the eigenvalue 1 (namely, $K[1]=1$ ).

$\left(A_{3}^{\prime}\right)$ The spectrum of $K$ (considered as an operator acting on $\left.L^{2}(\Omega)\right)$ is contained in the complex closed right-half plane.

The continuous version of Theorem 1.1 reads as follows.

Theorem 1.4. Assume $\left(A_{1}^{\prime}\right),\left(A_{2}^{\prime}\right)$ and $\left(A_{3}^{\prime}\right)$. Then, for any $c \in \mathbb{R}, 1$ is the unique bounded solution $p$ of 4 such that $\inf _{\mathbb{R} \times \Omega} p>0$.

We deduce just as before the uniqueness of the stationary states and the uniform convergence to the unique stationary state in the wake of the waves for (4), provided a uniform estimate from below can be shown. 
Corollary 1.5. Assume $\left(A_{1}^{\prime}\right),\left(A_{2}^{\prime}\right)$ and $\left(A_{3}^{\prime}\right)$. Then 1 is the unique bounded solution of (4) with positive infimum in $\mathbb{R} \times \Omega$ and with $c=0$.

Corollary 1.6. Assume $\left(A_{1}^{\prime}\right),\left(A_{2}^{\prime}\right)$ and $\left(A_{3}^{\prime}\right)$. Then all bounded classical solutions $(p, c)$ of (4) such that

$$
\lim _{\xi \rightarrow+\infty} \sup _{y \in \Omega} p(\xi, y)=0 \text { and } \liminf _{\xi \rightarrow-\infty} \inf _{y \in \Omega} p(\xi, y)>0
$$

actually satisfy

$$
\lim _{\xi \rightarrow-\infty} \sup _{y \in \Omega}|p(\xi, y)-1|=0 .
$$

1.3. Organization of the paper. In Section 2, we discuss the assumptions, the results and the literature. In Section 3, we prove Theorem 1.1. In Section 4, we prove Theorem 1.4

\section{Discussion}

2.1. The conditions on M. By definition, a matrix is line-sum-symmetric if the sum of coefficients in each of its rows equals the sum of coefficients in the corresponding column. Symmetric matrices and circulant matrices are line-sum-symmetric. Although the notions of symmetry, circulancy and line-sum-symmetry coincide in dimension 2, in dimension 3 and higher, there are line-sum-symmetric matrices that are neither symmetric nor circulant, as shown by the following counter-example:

$$
\left(\begin{array}{ccc}
a & 2 b & 0 \\
b & c & b \\
b & 0 & d
\end{array}\right) \quad \text { with } a, b, c, d \in \mathbb{R} .
$$

The study of line-sum-symmetric matrices was initiated by Eaves, Hoffman, Rothblum and Schneider 21]. Roughly speaking, these matrices conveniently generalize symmetric matrices when what we have in mind is summation of lines or rows of linear systems [21, Corollary 3], which is the case in this paper and more generally whenever we want to "integrate by parts" in a discrete variable. As such, they recently appeared in the literature on reaction-diffusion systems 15, 16.

2.1.1. The symmetric case. In the symmetric case, which arises in many applications, our assumption $\left(A_{1}\right)$ on $\mathbf{M}$ comes down to assuming that $\mathbf{M}$ has an "integration by parts" formula:

$$
\langle\mathbf{M u}, \mathbf{v}\rangle=-\frac{1}{2} \sum_{i, j \in[N]} m_{i, j}\left(u_{i}-u_{j}\right)\left(v_{i}-v_{j}\right) .
$$

where $\langle\cdot, \cdot\rangle$ is the canonical (Hermitian) scalar product on $\mathbb{C}^{N}$. A particularly natural example is the explicit Euler scheme for the one-dimensional heat equation with periodic boundary conditions: $\mathbf{M}=-\boldsymbol{\nabla}_{\mathrm{D}}^{\mathrm{T}} \boldsymbol{\Sigma} \boldsymbol{\nabla}_{\mathrm{D}}, \boldsymbol{\Sigma}=\operatorname{diag}\left(\sigma_{1}, \sigma_{2}, \ldots, \sigma_{N}\right)$ $\left(\sigma_{i}>0\right)$ and

$$
\nabla_{\mathrm{D}}=\left(\begin{array}{ccccccc}
-1 & 0 & 0 & \ldots & & & 1 \\
1 & -1 & 0 & 0 & \ldots & \ldots & 0 \\
0 & 1 & -1 & 0 & 0 & \ldots & \vdots \\
\vdots & \vdots & \vdots & \vdots & \vdots & & \vdots \\
0 & 0 & \cdots & 0 & & 1 & -1
\end{array}\right)
$$


The expanded form of $\mathbf{M}$ is

$$
\left.\begin{array}{cccccc}
-\sigma_{1}-\sigma_{2} & \sigma_{2} & 0 & \ldots & 0 & \sigma_{1} \\
\sigma_{2} & -\sigma_{2}-\sigma_{3} & \sigma_{3} & 0 & \ldots & 0 \\
0 & \sigma_{3} & -\sigma_{3}-\sigma_{4} & \sigma_{4} & 0 & \ldots \\
\vdots & \vdots & \vdots & \vdots & \vdots & \vdots \\
\sigma_{1} & 0 & \cdots & 0 & \sigma_{N} & -\sigma_{N}-\sigma_{1}
\end{array}\right) \quad \text { if } N \geq 3
$$

Neumann boundary conditions can be obtained by replacing the first line in $\nabla_{\mathrm{D}}$ by zero and also satisfy $\left(A_{1}\right)$. On the contrary, Dirichlet boundary conditions are qualitatively different (in particular, 1 cannot be a solution anymore) and are therefore outside the scope of this paper. Note that non-tridiagonal matrices can also be obtained in the form $-\nabla_{\mathrm{D}}^{\mathrm{T}} \boldsymbol{\Sigma} \boldsymbol{\nabla}_{\mathrm{D}}$ by allowing $\boldsymbol{\nabla}_{\mathrm{D}}$ to be non-square: as an example, discretization of divergence-form operators in two-dimensional domain such as

$$
\mathbf{M}=\left(\begin{array}{ccccc}
-\sigma_{1}-\sigma_{5} & 0 & \sigma_{1} & 0 & \sigma_{5} \\
0 & -\sigma_{2}-\sigma_{6} & \sigma_{2} & \sigma_{6} & 0 \\
\sigma_{1} & \sigma_{2} & -\sigma_{1}-\sigma_{2}-\sigma_{3}-\sigma_{4} & \sigma_{3} & \sigma_{4} \\
0 & \sigma_{6} & \sigma_{3} & -\sigma_{3}-\sigma_{6} & 0 \\
\sigma_{5} & 0 & \sigma_{4} & 0 & -\sigma_{4}-\sigma_{5}
\end{array}\right)
$$

are not always tridiagonal. In this case $\nabla_{\mathrm{D}} \in \mathscr{M}_{10,5}(\mathbb{R})$ corresponds to a discrete gradient operator on a cell with four boundary points and one interior point, and $\boldsymbol{\Sigma} \in \mathscr{M}_{10,10}(\mathbb{R})$ encodes the diffusion rates.

In addition to the divergence-form differential part presented above, $\mathbf{M}$ might also contain the discretization of a nonlocal integral operator, as hinted by (4).

2.2. The conditions on $\mathbf{C}$. The assumption that the Perron-Frobenius eigenvalue of $\mathbf{C}$ is unitary $\left(\lambda_{\mathrm{PF}}(\mathbf{C})=1\right)$ is done without loss of generality (up to replacing $(\mathbf{p}, \mathbf{C})$ by $\left.\left(\lambda_{\mathrm{PF}}(\mathbf{C}) \mathbf{p}, \lambda_{\mathrm{PF}}(\mathbf{C})^{-1} \mathbf{C}\right)\right)$. However the assumption that $\mathbf{1}$ is a PerronFrobenius eigenvector is a true assumption, not satisfied in general.

The set of real positive normal matrices contains as particular subsets the set of real positive circulant matrices and the set of real positive symmetric matrices (skew-symmetric and orthogonal matrices are normal but cannot be positive). The following counter-example shows that there are matrices satisfying $\left(A_{2}\right)$ and $\left(A_{3}\right)$ that are neither symmetric nor circulant:

$$
\left(\begin{array}{llll}
a & b & c & d \\
b & a & d & c \\
d & c & a & b \\
c & d & b & a
\end{array}\right) \quad \text { with } a, b, c, d>0, a+b+c+d=1
$$

(The eigenvalues of this matrix are $1, a+b-c-d, a-b \pm \mathrm{i}|c-d|$ and therefore $\left(A_{3}\right)$ is satisfied as soon as $a \geq b$ and $a+b \geq c+d$.)

In fact, a polynomial in any permutation matrix is normal. It is therefore possible to construct such counterexamples in any dimension $N \geq 4$, by selecting a permutation matrix associated with a cycle of maximal length which is not a power of the circular permutation.

2.2.1. The circulant case. In the circulant case, which is of particular interest to us, there exists a positive vector $\phi \in \mathbb{R}^{N}$ such that the matrix $\mathbf{C}$ is written as 
$\mathbf{C}=\left(\phi_{i-j}\right)_{i, j \in[N]}, \boldsymbol{\phi}$ being periodically extended by

$$
\phi_{i-j}= \begin{cases}\phi_{i-j}, & \text { if } i-j \geq 1 \\ \phi_{N+i-j}, & \text { if } i-j \leq 0\end{cases}
$$

The expanded form of $\mathbf{C}$ is then

$$
\left(\begin{array}{cccc}
\phi_{N} & \phi_{N-1} & \ldots & \phi_{1} \\
\phi_{1} & \phi_{N} & \ldots & \phi_{2} \\
\vdots & \vdots & \vdots & \vdots \\
\phi_{N-1} & \phi_{N-2} & \ldots & \phi_{N}
\end{array}\right)
$$

and the product $\mathbf{C u}$ can be rewritten as $\phi \star \mathbf{u}$, where $\star$ is the discrete circular convolution operator:

$$
(\phi \star \mathbf{u})_{i}=\sum_{j=1}^{N} \phi_{i-j} u_{j}
$$

Defining the normalized discrete Fourier transform matrix as

$$
\mathbf{U}_{\mathrm{DFT}}=\frac{1}{\sqrt{N}}\left(\exp \left(-\frac{2 \mathrm{i} \pi}{N}(j-1)(k-1)\right)\right)_{j, k \in[N]},
$$

we find that $\mathbf{U}_{\mathrm{DFT}}=\mathbf{U}$ for any circulant matrix $\mathbf{C}$. In particular, $\mathbf{1}$ is automatically a Perron-Frobenius eigenvector (and the normalization $\lambda_{\mathrm{PF}}(\mathbf{C})=1$ reads $\left.\sum_{i=1}^{N} \phi_{i}=1\right)$. Moreover, the following equalities hold true:

$$
\mathbf{U} \mathbf{C} \mathbf{U}^{-1} \mathbf{U} \mathbf{u}=\mathbf{U} \mathbf{C u}=\mathbf{U}(\boldsymbol{\phi} \star \mathbf{u})=\sqrt{N}(\mathbf{U} \phi) \circ(\mathbf{U u}) .
$$

It follows easily that the spectrum of $\mathbf{C}$ is contained in the closed right-half plane if and only if $\mathbf{U} \phi$, namely the discrete Fourier transform of $\phi$, is valued in the closed right-half plane.

Last, we point out additional alternative writings of the reaction term:

$$
\mathbf{u}-(\mathbf{C u}) \circ \mathbf{u}=\mathbf{u} \circ(\mathbf{1}-\phi \star \mathbf{u})=-\mathbf{u} \circ(\phi \star(\mathbf{u}-\mathbf{1})),
$$

2.3. The case $N=2$. In the case $N=2$, the matrices $\mathbf{M}$ and $\mathbf{C}$ can be rewritten as depending on two parameters only:

$$
\mathbf{M}=\left(\begin{array}{cc}
-\sigma & \sigma \\
\sigma & -\sigma
\end{array}\right), \quad \mathbf{C}=\left(\begin{array}{cc}
1-\gamma & \gamma \\
\gamma & 1-\gamma
\end{array}\right),
$$

where $\sigma>0$ and $\gamma \in(0,1)$. The linear stability of $\mathbf{1}$ can be decided by computing the eigenvalues $\lambda_{ \pm}^{\mathbf{M}-\mathbf{C}}$ of the matrix $\mathbf{M}-\mathbf{C}$,

$$
\lambda_{ \pm}^{\mathbf{M}-\mathbf{C}}=-1-(\gamma-\sigma) \pm|\gamma-\sigma|
$$

while the eigenvalues of $\mathbf{C}$ are $\lambda_{1}^{\mathbf{C}}=1$ and $\lambda_{-}^{\mathbf{C}}=1-2 \gamma$. Therefore, $\mathbf{M}-\mathbf{C}$ has always one negative eigenvalue $\lambda_{-}^{\mathbf{M}-\mathbf{C}}<0$ and the behavior of $\lambda_{+}^{\mathbf{M}-\mathbf{C}}$ depends on the value of $\gamma$ :

a) if $\gamma \in(0,1 / 2)$ (in which case $\left(A_{3}\right)$ holds), $\lambda_{1}^{\mathbf{M}-\mathbf{C}}$ always stays negative,

b) if $\gamma \in(1 / 2,1)$ (in which case $\left(A_{3}\right)$ does not hold),

$$
\begin{aligned}
& \lambda_{+}^{\mathbf{M}-\mathbf{C}}>0 \text { if } 0<\sigma<\sigma^{*}:=\gamma-\frac{1}{2}, \\
& \lambda_{+}^{\mathbf{M}-\mathbf{C}}<0 \text { if } \sigma>\sigma^{*} .
\end{aligned}
$$

In the latter case, using $\sigma$ as a bifurcation parameter, a local bifurcation is occurring when decreasing $\sigma$ below $\sigma^{*}$ and two stable equilibria emerge when $\mathbf{1}$ loses stability. In particular, in this case there are solutions to (2) other than the constant 1 which are bounded from below. This is confirmed by the result in [17, Proposition 3.4]. 
2.4. KPP systems. The system (1) is a particular example of KPP systems. The first author studied these systems in $24-27]$. The second author studied them with collaborators in 3,29 and gave an epidemiological interpretation in 30 . Other important mathematical references are $5,17,19,31$. For a detailed overview of the literature, we refer to 25 .

These nonlinear, non-variational and non-cooperative (or non-monotone, in other words devoid of comparison principle) reaction-diffusion systems are referred to as "KPP systems" due to their structural similarity with the scalar Fisher-KPP equation,

$$
\partial_{t} u-\partial_{x x} u=u(1-u)
$$

(This scalar equation can actually be understood as a KPP system of dimension 1.) This similarity mainly concerns the behavior close to $\mathbf{u}=\mathbf{0}$ and it leads to several classical results: a sharp persistence-extinction criterion [25, 26], the existence of traveling waves for all speeds larger than or equal to a linearly determined minimal wave speed $c^{\star} 25,29,31$, the equality between this minimal wave speed and the asymptotic speed of spreading for initially compactly supported solutions of the Cauchy problem 5, 25] and an exponential equivalent of the profile at the leading edge 24,31.

However, away from $\mathbf{u}=\mathbf{0}$ and in particular in the wake of a traveling wave solution $\mathbf{p}(x-c t)$, the picture is more complicated. For two-component systems, locally uniform convergence of the solutions of the Cauchy problem to a unique constant steady state can be proved in many cases (and directly implies the convergence in the wake of the traveling waves) [27, Appendix B], [29], but bistable cases (corresponding to strongly competitive systems with weak mutations) still exist 17, 24 and remain elusive - in particular, traveling waves connecting $\mathbf{0}$ to an unstable constant steady state exist in some particular bistable cases [24]. For systems of any size but where $\mathbf{D}=\mathbf{I}$ and $\mathbf{C}=\mathbf{1 a}^{\mathrm{T}}$, locally uniform convergence of the solutions of the Cauchy problem to a unique constant steady state can be established 24], but these assumptions are in fact so strong that the system is basically reduced to a scalar Fisher-KPP equation projected along the Perron-Frobenius eigenvector of the linear part of the reaction term. More recent results confirm that, as soon as there are at least three components, convergence fails in general. In particular, for circulant matrices $\mathbf{M}$ and $\mathbf{C}$, Hopf bifurcations can occur and these typically lead to the formation of limit cycles, periodic wave trains, pulsating traveling waves and propagating terraces [27].

In this regard, the main result of this paper provides some sufficient conditions to prevent the formation of these oscillations in the elliptic and traveling wave problems. In the class of pairs $(\mathbf{M}, \mathbf{C})$ satisfying $\left(A_{1}\right)$ and $\left(A_{2}\right)$ the sharpness of $\left(A_{3}\right)$ (the spectrum of $\mathbf{C}$ is in the right-half plane) can be discussed as follows:

- in view of the Hopf-bifurcating case in 27], the system can be oscillatory if $\mathbf{C}$ admits an eigenvalue with negative real part and nonzero imaginary part;

- in view of the two-component case discussed in Section 2.3 (see also 17 Proposition 3.4]), there can be a multiplicity of positive constant equilibria when at least one eigenvalue of $\mathbf{C}$ is real and negative.

In the class of pairs $(\mathbf{M}, \mathbf{C})$ satisfying $\left(A_{2}\right),\left(A_{3}\right)$ and the mere irreducibility of $\mathbf{M}$, the sharpness of $\left(A_{1}\right)$ is unclear. The proof presented here heavily relies on the line-sum-symmetry of $\mathbf{M}$ and cannot be extended to more general matrices $\mathbf{M}$ (see Remark 3.1 below).

Let us point out that the convergence result here is strikingly new in the sense that it does not require the equality between all diffusion rates $(\mathbf{D}=\mathbf{I})$, which was required in 24,29]. The convergence results for two-component systems presented 
in 17. do not require such an assumption but use the boundedness of the domain to overcome this lack of structure; when extending these results to the unbounded setting, the equality $d_{1}=d_{2}$ is useful [27, Appendix B].

2.5. The nonlocal KPP equation. The spatially homogeneous system

$$
\dot{\mathbf{u}}=\mathbf{M u}+\mathbf{u} \circ(\mathbf{1}-\phi \star \mathbf{u})
$$

is, in a way, a discretized version of the nonlocal Fisher-KPP equation:

$$
\partial_{t} u=\partial_{x x} u+u(1-\phi \star u) .
$$

This nonlocal equation has attracted a lot of attention in the last few years. The existing literature (e.g., $1,7,12,22$, and references therein]) develops new techniques to overcome the default of comparison principle and these techniques proved to be fruitful when applied to non-cooperative KPP systems 25,29$]$. In the present paper we will once again import such a technique from 7 .

2.6. The nonlocal cane-toad equation. The diffusive system (1) is, in a similar way, a discretized version of the nonlocal cane-toad equation:

$$
\left\{\begin{array}{l}
\partial_{t} u=d(\theta) \partial_{x x} u+\alpha \partial_{\theta \theta} u+u(t, x, \theta)\left(1-\frac{1}{\bar{\theta}-\underline{\theta}} \int_{\theta=\underline{\theta}}^{\bar{\theta}} u\left(t, x, \theta^{\prime}\right) \mathrm{d} \theta^{\prime}\right), \\
\partial_{\theta} u(t, x, \underline{\theta})=\partial_{\theta} u(t, x, \bar{\theta})=0
\end{array}\right.
$$

where $u(t, x, \theta)$ is a population density structured with respect to a phenotypic trait $\theta \in[\underline{\theta}, \bar{\theta}] \subset[0,+\infty]$. This eco-evolutionary model has also attracted attention recently (e.g., $2,4,6,8,11,14,28,32]$ ), especially due to an acceleration phenomenon when $d(\theta)=\theta$ and $\theta=+\infty$ but also because, just like the nonlocal KPP equation, it does not satisfy the comparison principle and requires new techniques.

It turns out that the similarity between our system and this equation is so strong that our proof can be readily adapted and our result extends to this continuous-trait model (see Theorem 1.4 and its two corollaries).

2.7. More general reaction terms. In the system (1), the reaction term has the form $(\mathbf{I}+\mathbf{M}) \mathbf{u}-\mathbf{u} \circ(\mathbf{C u})$. It is natural to try to extend the results to reaction terms of the form $(\operatorname{diag}(\mathbf{r})+\mathbf{M}) \mathbf{u}-\mathbf{u} \circ(\mathbf{C u})$, where $\operatorname{diag}(\mathbf{r})+\mathbf{M}$ has a positive Perron-Frobenius eigenvalue, or $(\operatorname{diag}(\mathbf{r})+\mathbf{M}) \mathbf{u}-(\operatorname{diag}(\mathbf{r}) \mathbf{u}) \circ(\mathbf{C u})$, where $\mathbf{r}$ is positive. However our proof does not easily extend to such cases. The Liouville-type result for such cases remains as an open problem.

2.8. The Cauchy problem. It would be natural to try to prove that, with the same assumptions $\left(A_{1}\right)+\left(A_{3}\right)$ or $\left(A_{1}^{\prime}\right)+\left(A_{3}^{\prime}\right)$ the solutions of the parabolic Cauchy problem converge locally uniformly to $\mathbf{1}$. In fact, what can be proved, using the same test function $\left(\left(u_{i}-1\right) / u_{i}\right)_{i \in[N]}$ and the same calculations, is the locally uniform convergence to $\mathbf{1}$ under the additional assumption $\mathbf{D}=\mathbf{I}$. It is indeed wellknown that such an assumption makes it possible to use convex Lyapunov functions for parabolic systems $[33]$; in the present case, the convex Lyapunov function is $\mathbf{u}-\ln \mathbf{u}$ (where, obviously, the notation $\ln \mathbf{u}$ stands for $\left.\left(\ln u_{i}\right)_{i \in[N]}\right)$. See also 20 , Section 7] for a similar argument.

The locally uniform convergence when $\mathbf{D} \neq \mathbf{I}$ remains as an open problem.

\section{Proof of Theorem 1.1}

Our strategy is to mimic the proof of 7 . Theorem 4.1], which uses the test function $(p-1) / p$. More precisely, we rely upon

$$
\sum_{i=1}^{N} \frac{(\mathbf{M} \mathbf{p})_{i}}{p_{i}} \geq 0 \quad \text { with equality iff } \mathbf{p} \in \operatorname{span}(\mathbf{1}),
$$


and upon

$$
\sum_{i=1}^{N}\left(p_{i}-1\right)(\mathbf{C}(\mathbf{p}-\mathbf{1}))_{i} \geq 0 .
$$

The inequality (5) is a standard property of irreducible line-sum-symmetric matrices (recalled in the forthcoming Lemma 3.1); the inequality (6) is a direct consequence of (a generalized version of) Plancherel's theorem:

$$
\begin{aligned}
\sum_{i=1}^{N} q_{i}(\mathbf{C q})_{i} & =\operatorname{Re}\left(\sum_{i=1}^{N} q_{i}(\mathbf{C q})_{i}\right) \\
& =\operatorname{Re}(\langle\mathbf{q}, \mathbf{C q}\rangle) \\
& =\operatorname{Re}\left(\left\langle\mathbf{q}, \overline{\mathbf{U}}^{\mathrm{T}} \mathbf{U} \mathbf{C} \overline{\mathbf{U}}^{\mathrm{T}} \mathbf{U} \mathbf{q}\right\rangle\right) \\
& =\operatorname{Re}\left(\left\langle\mathbf{U} \mathbf{q}, \mathbf{U} \mathbf{C} \overline{\mathbf{U}}^{\mathrm{T}} \mathbf{U} \mathbf{q}\right\rangle\right) \\
& \geq \min _{\lambda \in \operatorname{sp}(\mathbf{C})}(\operatorname{Re}(\lambda)) \sum_{i=1}^{N}\left|(\mathbf{U q})_{i}\right|^{2},
\end{aligned}
$$

where $\langle\cdot, \cdot\rangle$ is the canonical (Hermitian) scalar product on $\mathbb{C}^{N}$.

Lemma 3.1 (Classification of line-sum-symmetric matrices [21, Corollary 3]). Let $\mathbf{A} \in \mathscr{M}_{N, N}(\mathbb{R})$ be a nonnegative matrix. Then $\mathbf{A}$ is line-sum-symmetric if and only if

$$
\sum_{i, j \in[N]} \frac{a_{i, j} u_{j}}{u_{i}} \geq \sum_{i, j \in[N]} a_{i, j} \quad \text { for all } \mathbf{u} \in(0,+\infty)^{N} .
$$

Furthermore, if $\mathbf{A}$ is irreducible and line-sum-symmetric, equality above holds if and only if $\mathbf{u} \in \operatorname{span}(\mathbf{1})$.

The inequality (5) follows then from Lemma 3.1 applied to the nonnegative, line-sum-symmetric and irreducible matrix $\mathbf{A}=\mathbf{M}-\min _{i \in[N]}\left(m_{i, i}\right) \mathbf{I}$.

Lemma 3.2 (Uniqueness of the nonzero constant solution). The unique nonnegative nonzero solution of $\mathbf{M u}+\mathbf{u}=(\mathbf{C u}) \circ \mathbf{u}$ is $\mathbf{1}$.

Proof. Let $\mathbf{u}$ be any nonnegative nonzero solution. Recall that $\mathbf{u}$ is in fact positive. Denoting $\mathbf{u}^{\circ-1}=\left(1 / u_{i}\right)_{i \in[N]}$ and taking the scalar product

$$
\left\langle-\mathbf{M u}-\mathbf{u} \circ(\mathbf{1}-\mathbf{C u}), \mathbf{u}^{\circ-1} \circ(\mathbf{u}-\mathbf{1})\right\rangle=\mathbf{0},
$$

we get

$$
-\left\langle\mathbf{M u}, \mathbf{u}^{\circ-1}\right\rangle=\langle\mathbf{C}(\mathbf{u}-\mathbf{1}), \mathbf{u}-\mathbf{1}\rangle .
$$

On one hand, by (5), the left-hand side is nonpositive. On the other hand, by (6), the right-hand side is nonnegative. Therefore both sides are zero. Using now the case of equality in (5), we deduce $\mathbf{u} \in \operatorname{span}(\mathbf{1})$. We deduce subsequently from the right-hand side that $\mathbf{u}=\mathbf{1}$.

We are now in a position to prove Theorem 1.1

Proof of Theorem 1.1. Let $(\mathbf{p}, c)$ be a bounded solution of the system (2) satisfying $\inf _{\mathbb{R}} p_{i}>0$ for any $i \in[N]$.

Step 1: We show that

$$
\lim _{\xi \rightarrow \pm \infty} \mathbf{p}(\xi)=\mathbf{1}
$$


At any $\xi \in \mathbb{R}$, denoting $\mathbf{p}^{\circ-1}(\xi)=\left(1 / p_{i}(\xi)\right)_{i \in[N]}$ and taking the scalar product (in $\mathbb{R}^{N}$ )

$$
\left\langle-\mathbf{D} \mathbf{p}^{\prime \prime}-c \mathbf{p}^{\prime}-\mathbf{M} \mathbf{p}-\mathbf{p} \circ(\mathbf{1}-\mathbf{C p}), \mathbf{p}^{\circ-1} \circ(\mathbf{p}-\mathbf{1})\right\rangle=\mathbf{0},
$$

we get

$$
\sum_{i=1}^{N}\left[-\left(d_{i} p_{i}^{\prime \prime}+c p_{i}^{\prime}\right)\left(\frac{p_{i}-1}{p_{i}}\right)\right]=-\sum_{i=1}^{N} \frac{(\mathbf{M} \mathbf{p})_{i}}{p_{i}}-\sum_{i=1}^{N}\left(p_{i}-1\right)(\mathbf{C}(\mathbf{p}-\mathbf{1}))_{i} .
$$

By (5) and (6), the right-hand side is nonpositive and therefore

$$
\sum_{i=1}^{N}\left[-\left(d_{i} p_{i}^{\prime \prime}+c p_{i}^{\prime}\right)\left(\frac{p_{i}-1}{p_{i}}\right)\right] \leq 0
$$

Since this holds true at any $\xi \in \mathbb{R}$, we fix $R>0$ and integrate by parts in $[-R, R]$. We get, as in [7, Proof of Lemma 4.1],

$$
\sum_{i=1}^{N} d_{i} \int_{-R}^{R}\left(\frac{p_{i}^{\prime}}{p_{i}}\right)^{2} \leq \sum_{i=1}^{N}\left[d_{i} \frac{p_{i}^{\prime}\left(p_{i}-1\right)}{p_{i}}+c \ln \left(p_{i}\right)-c p_{i}\right]_{-R}^{R},
$$

By the classical elliptic estimates, $\left|p_{i}^{\prime}( \pm R)\right|$ is bounded by $\max _{i \in[N]} \sup _{\mathbb{R}} p_{i}$ up to a multiplicative constant independent of $R$. Recalling that $p_{i}$ is uniformly bounded from below by $\min _{i \in[N]} \inf _{\mathbb{R}} p_{i}>0$, the right-hand side is bounded by a constant independent of $R$. We deduce that $p_{i}^{\prime} \in L^{2}(\mathbb{R})$ for all $i \in[N]$.

Let now $\left(\xi_{n}\right)_{n \in \mathbb{N}}$ be any sequence such that $\xi_{n} \rightarrow-\infty$ and define $\mathbf{p}^{n}: \xi \mapsto$ $\mathbf{p}\left(\xi+\xi_{n}\right)$. We remark that, for all $i \in[N]$ and all $\zeta \in \mathbb{R}$, we have

$$
\int_{-\infty}^{\zeta}\left[\left(p_{i}^{n}\right)^{\prime}\right]^{2}(\xi) \mathrm{d} \xi=\int_{-\infty}^{\zeta+\xi_{n}}\left(p_{i}^{\prime}\right)^{2}(\xi) \mathrm{d} \xi \underset{n \rightarrow+\infty}{\longrightarrow} 0 \text { for all } i \in[N],
$$

and therefore $\left(\mathbf{p}^{n}\right)^{\prime}$ converges to $\mathbf{0}$ locally uniformly in $L^{2}$. Next, using the classical elliptic estimates, we extract from $\left(\mathbf{p}^{n}\right)_{n \in \mathbb{N}}$ a subsequence which converges in $\mathscr{C}_{\text {loc }}^{2}$ to a limit $\mathbf{p}^{\infty} \in \mathscr{C}^{2}(\mathbb{R})$. Note that $\mathbf{p}^{\infty}$ is still a solution to $(2)$. Since $\left(\mathbf{p}^{n}\right)^{\prime} \rightarrow 0$ in $L_{\text {loc }}^{2}$, we conclude that $\mathbf{p}^{\infty}$ has to be a constant function of the variable $\xi$, i.e. a constant solution of $\mathbf{M p}+\mathbf{p}=(\mathbf{C p}) \circ \mathbf{p}$. By Lemma $3.2 \mathbf{p}^{\infty}=\mathbf{1}$ identically.

Since the sequence $\left(\xi_{n}\right)_{n \in \mathbb{N}}$ is arbitrary, we have shown that

$$
\lim _{\xi \rightarrow-\infty} \mathbf{p}(\xi)=\mathbf{1}
$$

The limit at $+\infty$ can be established by a similar argument.

Step 2: We show that $\mathbf{p}$ is identically equal to $\mathbf{1}$. Since $\mathbf{p}$ converges to $\mathbf{1}$ on both sides of the real line, the brackets on the right-hand side of 7 converge to 0 as $R \rightarrow+\infty$. Therefore,

$$
\begin{aligned}
0 \leq \sum_{i=1}^{N} \int_{-\infty}^{+\infty}\left(p_{i}^{\prime}\right)^{2} & =\lim _{R \rightarrow+\infty} \sum_{i=1}^{N} \int_{-R}^{R}\left(p_{i}^{\prime}\right)^{2} \\
& \leq \lim _{R \rightarrow+\infty} \frac{\max _{i \in[N]} \sup _{\mathbb{R}} p_{i}^{2}}{\min _{i \in[N]} d_{i}} \sum_{i=1}^{N} d_{i} \int_{-R}^{R}\left(\frac{p_{i}^{\prime}}{p_{i}}\right)^{2} \\
& \leq C \lim _{R \rightarrow+\infty} \sum_{i=1}^{N}\left[d_{i} \frac{p_{i}^{\prime}\left(p_{i}-1\right)}{p_{i}}+c \ln \left(p_{i}\right)-c p_{i}\right]_{-R}^{R}=0
\end{aligned}
$$

where $C$ is a constant independent of $R$. We conclude that $\mathbf{p}$ has to be a constant function of $\xi$, and the only possibility is $\mathbf{p}=\mathbf{1}$. 
Remark 3.1. From the proofs of Lemma 3.2 and Theorem 1.1 above, it is clear that the estimate $\left\langle\mathbf{u}^{\circ-1}, \mathbf{M u}\right\rangle \geq 0$, together with the equality case, is crucial. Since this estimate fails if $\mathbf{M}$ is not line-sum-symmetric (by Lemma 3.1), $\left(A_{1}\right)$ is sharp regarding the proof presented here. Note that [21, Theorem 2] proved that every nonnegative irreducible matrix $\mathbf{A}$ has a line-sum-symmetric similarity-scaling $\operatorname{diag}(\mathbf{x}) \mathbf{A} \operatorname{diag}(\mathbf{x})^{-1}$, where $\mathbf{x}$ is a positive vector, but it seems to us that this property cannot be used to generalize the above proof to non-line-sum-symmetric matrices $\mathbf{M}$.

Finally we recall briefly the arguments leading to the proof of Corollary 1.2 and 1.3

Sketch of the proof of Corollary 1.2. Let $\mathbf{p}$ be a bounded steady state solution of (1), i.e. a traveling wave with speed $c=0$. It is known from [25. Theorem 1.3 (ii)] that, if $\mathbf{p}$ is nonnegative and nonzero, then $\mathbf{p}$ is bounded uniformly away from $\mathbf{0}$. Theorem 1.1 concludes.

Sketch of the proof of Corollary 1.3. Let $(\mathbf{p}, c)$ be a bounded traveling wave solution of (1) satisfying the boundary conditions (3). By [25, Theorem 1.5 (iii)], condition (3) near $-\infty$ immediately transfers to

$$
\min _{i \in[N]} \liminf _{\xi \rightarrow-\infty} p_{i}(\xi)>0,
$$

therefore Theorem 1.1 can be applied to any local uniform limit of a converging sequence $\mathbf{p}\left(\xi+\xi_{n}\right)$ for some $\xi_{n} \rightarrow-\infty$. Since the limit is uniquely identified, the claim is proved.

\section{Proof of Theorem 1.4}

We follow the same steps as for the proof of Theorem 1.1, but have to adapt each argument in the correct functional setting.

Lemma 4.1 (Positivity of $K)$. Assume $\left(A_{2}^{\prime}\right)$ and $\left(A_{3}^{\prime}\right)$. Then for all nonzero $u \in L^{2}(\Omega)$,

$$
\langle K[u], u\rangle_{L^{2}(\Omega)}=\int_{\Omega^{2}} u(y) k(y, z) u(z) \mathrm{d} y \mathrm{~d} z \geq 0 .
$$

Proof. To prove the result, we take advantage of the spectral decomposition of $K$ considered as an operator acting on the complex Hilbert space $L_{\mathbb{C}}^{2}(\Omega)$ equipped with the canonical hermitian product $\langle f, g\rangle_{L_{\mathbb{C}}^{2}}=\int_{\Omega} f \bar{g}$. Clearly $K$ is still normal when considered as an operator on $L_{\mathbb{C}}^{2}(\Omega)$. Moreover, by Lemma $4.4, K$ is compact (and the compactness classically transfers to the complex extension of $K)$. Since $L_{\mathbb{C}}^{2}(\Omega)$ is separable, by the spectral decomposition theorem (see e.g. 13 . Proposition 11.36 p.369]), there exists a Hilbert basis of $L_{\mathbb{C}}^{2}(\Omega)$ composed of eigenvectors of $K$. Let us denote $\left(e_{n}\right)_{n \in \mathbb{N}}$ such a Hilbert basis and $\left(\lambda_{n}\right)_{n \in \mathbb{N}}$ the corresponding sequence of eigenvalues. This decomposition yields

$$
\langle K[u], u\rangle_{L_{\mathbb{C}}^{2}}=\sum_{n=0}^{+\infty} \lambda_{n}\left|\left\langle u, e_{n}\right\rangle_{L_{\mathbb{C}}^{2}}\right|^{2},
$$

but since $\langle K[u], u\rangle_{L_{\mathbb{C}}^{2}}$ is real,

$$
\langle K[u], u\rangle_{L_{\mathbb{C}}^{2}}=\operatorname{Re}\left(\sum_{n=0}^{+\infty} \lambda_{n}\left|\left\langle u, e_{n}\right\rangle_{L_{\mathbb{C}}^{2}}\right|^{2}\right)=\sum_{n=0}^{+\infty} \operatorname{Re}\left(\lambda_{n}\right)\left|\left\langle u, e_{n}\right\rangle_{L_{\mathbb{C}}^{2}}\right|^{2} \geq 0 .
$$

Lemma 4.2 (Characterization of continuous line-sum-symmetric operators 16 , Theorem 4]). Let $a \in \mathscr{C}(\Omega \times \Omega,[0,+\infty))$ be Riemann integrable. Then the following two properties are equivalent: 
(1) $\int_{\Omega} a(x, y) \mathrm{d} y=\int_{\Omega} a(y, x) \mathrm{d} y$ for all $x \in \Omega$;

(2) $\int_{\Omega \times \Omega} \frac{a(x, y) u(y)}{u(x)} \mathrm{d} y \mathrm{~d} x \geq \int_{\Omega \times \Omega} a(x, y) \mathrm{d} y \mathrm{~d} x$ for all $u \in \mathscr{C}(\bar{\Omega},(0,+\infty))$.

We point out that the equality case of the second property is not presented in the above lemma but was studied in [16. Theorem 5] under the irreducibility-type assumption that $(x, y) \mapsto a(x, y)+a(y, x)$ does not vanish. Here, we need in any case to include in the mutation operator a nontrivial divergence part $(\sigma>0)$, and this suffices for the irreducibility-type properties we need, so that we do not make any irreducibility-type assumption on the nonlocal part.

Lemma 4.3 (Uniqueness of the constant solution). Assume $\left(A_{1}^{\prime}\right),\left(A_{2}^{\prime}\right)$ and $\left(A_{3}^{\prime}\right)$. The constant 1 is the unique nonnegative nonzero classical solution to the equation

$$
\nabla_{y} \cdot\left(\sigma(y) \nabla_{y} p\right)+M[p](y)+p(y)(1-K[p](y))=0,
$$

supplemented with homogeneous Neumann boundary conditions on $\partial \Omega$.

Proof. We first remark that, by a direct application of the strong maximum principle and Hopf's lemma, the fact that $p$ is nonzero can be reinforced as $p(y)>0$ on $\bar{\Omega}$. Since moreover $p$ is continuous on $\bar{\Omega}, p$ is bounded from below. In particular, the test function $\frac{p(y)-1}{p(y)}$ is well-defined and (at least) in $\mathscr{C}^{1}(\bar{\Omega})$.

As in the discrete case, we multiply $(8)$ by $\frac{p(y)-1}{p(y)}$ and integrate over $\Omega$. Integrating by parts the gradient term, we get:

$$
\begin{aligned}
0= & -\int_{\Omega} \sigma(y) \nabla_{y} p(y) \nabla_{y}\left(1-\frac{1}{p(y)}\right) \mathrm{d} y+\int_{\Omega \times \Omega} m(y, z)(p(z)-p(y)) \mathrm{d} z \mathrm{~d} y \\
& -\int_{\Omega \times \Omega} m(y, z)(p(z)-p(y)) \frac{1}{p(y)} \mathrm{d} z \mathrm{~d} y+\int_{\Omega}(1-K[p](y))(p(y)-1) \mathrm{d} y .
\end{aligned}
$$

Let us show that each of those terms is nonpositive. We first remark that

$$
\begin{aligned}
-\int_{\Omega} \sigma(y) \nabla_{y} p(y) \nabla_{y} & \left(1-\frac{1}{p(y)}\right) \mathrm{d} y=-\int_{\Omega} \sigma(y) \frac{|\nabla p|^{2}}{p(y)^{2}} \mathrm{~d} y \leq 0, \\
\int_{\Omega \times \Omega} m(y, z)(p(z)-p(y)) \mathrm{d} z \mathrm{~d} y & =\int_{\Omega}\left(\int_{\Omega} m(y, z) p(z) \mathrm{d} z-\int_{\Omega} m(y, z) \mathrm{d} z p(y)\right) \mathrm{d} y \\
& =\int_{\Omega}\left(\int_{\Omega} m(y, z) p(z) \mathrm{d} z-\int_{\Omega} m(z, y) \mathrm{d} z p(y)\right) \mathrm{d} y \\
& =\int_{\Omega \times \Omega} m(y, z) p(z) \mathrm{d} z \mathrm{~d} y-\int_{\Omega \times \Omega} m(z, y) p(y) \mathrm{d} z \mathrm{~d} y \\
& =0 .
\end{aligned}
$$

Next, by Lemma 4.2 .

$$
\int_{\Omega \times \Omega} m(y, z)(p(z)-p(y)) \frac{1}{p(y)} \mathrm{d} y \mathrm{~d} z=\int_{\Omega \times \Omega} \frac{m(y, z) p(z)}{p(y)} \mathrm{d} y \mathrm{~d} z-\int_{\Omega \times \Omega} m \geq 0 .
$$

Finally, since $K[1]=1$, we have $1-K[p]=K[1-p]$ and thus, by Lemma 4.1

$$
\int_{\Omega}(1-K[p](y))(p(y)-1) \mathrm{d} y=-\int_{\Omega} K[1-p](y)(1-p(y)) \mathrm{d} y \leq 0 .
$$

Therefore each of those four terms is in fact equal to 0 . From $\int_{\Omega} \sigma(y) \frac{|\nabla p(y)|^{2}}{p(y)^{2}} \mathrm{~d} y=$ 0 we deduce that $p(y)$ is a constant on $\bar{\Omega}$. Since then

$$
0=\int_{\Omega} K[1-p](1-p) \mathrm{d} y=(1-p)^{2} \int_{\Omega^{2}} k(y, z) \mathrm{d} y \mathrm{~d} z
$$

and $\int_{\Omega^{2}} k(y, z) \mathrm{d} y \mathrm{~d} z>0$, we conclude that $p=1$. 
We are now in a position to prove Theorem 1.4

Proof of Theorem 1.4. As in the discrete case (proof of Theorem 1.1), we multiply the equation (4) by the test function $\frac{p(\xi, y)-1}{p(\xi, y)}$ and integrate on the cylinder $\Omega_{R}=$ $[-R, R] \times \Omega$ for some $R>0$. With the exact same computations as in the proof of Lemma 4.3 we get

$$
-\int_{\Omega_{R}}\left(d(y) \partial_{\xi \xi} p(\xi, y)+c \partial_{\xi} p(\xi, y)\right) \frac{p(\xi, y)-1}{p(\xi, y)} \mathrm{d} \xi \mathrm{d} y \leq 0 .
$$

After integrations by parts in the $\xi$ variable, we find

$$
\text { (9) } \begin{aligned}
\int_{\Omega_{R}} d(y) \frac{\left|\partial_{\xi} p(\xi, y)\right|^{2}}{p(\xi, y)^{2}} \mathrm{~d} \xi \mathrm{d} y \\
\leq\left[\int_{\Omega} d(y) \frac{\partial_{\xi} p(\xi, y)(p(\xi, y)-1)}{p(\xi, y)}+c(\ln (p(\xi, y))-p(\xi, y)) \mathrm{d} y\right]_{-R}^{R}
\end{aligned}
$$

where, by the classical elliptic estimates, $\left|\partial_{\xi} p( \pm R, y)\right|$ is controlled from above by $\sup _{(\xi, y) \in \mathbb{R} \times \Omega} p(\xi, y)$, independently of $R$. Taking the limit $R \rightarrow+\infty$, we see that $\partial_{\xi} p(\xi, y) \in L^{2}(\mathbb{R} \times \Omega)$. Using elliptic regularity, a translation argument (which is similar to the one developed in the proof of Theorem 1.1) and Lemma 4.3, we conclude that

$$
\lim _{\xi \rightarrow \pm \infty} \sup _{y \in \Omega}|p(\xi, y)-1|=0 .
$$

Going back to (9), we easily see that the right-hand side converges to zero as $R \rightarrow+\infty$ and therefore

$$
\int_{\mathbb{R} \times \Omega} d(y) \frac{\left|\partial_{\xi} p(\xi, y)\right|^{2}}{p(\xi, y)^{2}} \mathrm{~d} \xi \mathrm{d} y=0
$$

thus $p$ is constant in $\xi$. In view of the limiting conditions, we conclude that in fact $p=1$ identically.

Corollary 1.5 is a direct application of Theorem 1.4 As for Corollary 1.6 it is proven by an argument similar to the one that yields the limit of the solution near $\pm \infty$ in the proof of Theorem 1.4. Since it is rather classical to adapt this argument for traveling waves, we omit the details.

We end by a technical but necessary lemma.

Lemma 4.4 (Compactness of $K$ ). Assume $\left(A_{2}^{\prime}\right)$. Then the operator $K: L^{2}(\Omega) \rightarrow$ $L^{2}(\Omega)$ is compact.

Proof. We aim at applying the Kolmogorov-Riesz-Fréchet Theorem (see e.g. 13 Theorem 4.26 p.111]) to our operator $K$. We extend the function $k(y, z)$ to $\mathbb{R}^{Q} \times \overline{\mathbb{R}}^{Q}$ by setting $k(y, z)=0$ for $y, z \notin \Omega$. For $f \in L^{2}\left(\mathbb{R}^{Q}\right)$ we define:

$$
K[f](y)=\int_{\mathbb{R}^{Q}} k(y, z) f(z) \mathrm{d} z .
$$

Let $\varepsilon>0$ and $f \in L^{2}(\Omega),\|f\|_{L^{2}(\Omega)}=1$ be given. We extend $f$ to $L^{2}\left(\mathbb{R}^{Q}\right)$ by setting $f(z)=0, z \notin \Omega$. We also define, for all $h \in \mathbb{R}^{Q}$, the translation operator 
$\tau_{h}: g \mapsto g(\bullet+h)$. We first remark that, for any $h \in \mathbb{R}^{Q}$,

$$
\begin{aligned}
\left\|\tau_{h} K[f]-K[f]\right\|_{L^{2}}^{2} & =\int_{\mathbb{R}^{Q}}\left(\int_{\mathbb{R}^{Q}} k(y+h, z) f(z) \mathrm{d} z-\int_{\mathbb{R}^{Q}} k(y, z) f(z) \mathrm{d} z\right)^{2} \mathrm{~d} y \\
& =\int_{\mathbb{R}^{Q}}\left(\int_{\mathbb{R}^{Q}}(k(y+h, z)-k(y, z)) f(z) \mathrm{d} z\right)^{2} \mathrm{~d} y \\
& \leq \int_{\mathbb{R}^{Q}} \int_{\mathbb{R}^{Q}}(k(y+h, z)-k(y, z))^{2} \mathrm{~d} z \mathrm{~d} y\|f\|_{L^{2}} \\
& =\int_{\mathbb{R}^{Q}} \int_{\mathbb{R}^{Q}}(k(y+h, z)-k(y, z))^{2} \mathrm{~d} z \mathrm{~d} y,
\end{aligned}
$$

where we have used the classical Cauchy-Schwarz inequality in $L^{2}\left(\mathbb{R}^{Q}\right)$. Therefore it only remains to control the $L^{2}$ norm of $k(y+h, \cdot)-k(y, \cdot)$ when $h$ is small. To this aim we fix $\delta_{1}>0$ be such that

$$
\left|\left\{d(y, \partial \Omega) \leq \delta_{1}\right\}\right| \leq \frac{\varepsilon}{8\|k\|_{L^{\infty}\left(\Omega^{2}\right)}^{2}|\Omega|},
$$

where $d(\cdot, \partial \Omega)$ is the Euclidean distance between $y \in \mathbb{R}^{Q}$ and the set $\partial \Omega$ and $\left|\left\{d(y, \partial \Omega) \leq \delta_{1}\right\}\right|$ is the Lebesgue measure of the set of points $y \in \mathbb{R}^{Q}$ satisfying $d(y, \partial \Omega) \leq \delta_{1}$. Since $k$ is continuous on the compact set $\bar{\Omega}^{2}$, there exists $\delta_{2}>0$ such that $|k(y+h, z)-k(y, z)| \leq \frac{\varepsilon}{\sqrt{2}|\Omega|}$ if $y, y+h, z \in \bar{\Omega}$ and $|h| \leq \delta_{2}$.

Therefore, if $|h| \leq \min \left(\delta_{1}, \delta_{2}\right)$, we have:

$$
\begin{aligned}
\left\|\tau_{h} K[f]-K[f]\right\|_{L^{2}}^{2}= & \int_{d(y, \partial \Omega) \leq \delta_{1}} \int_{\mathbb{R}^{Q}}(k(y+h, z)-k(y, z))^{2} \mathrm{~d} z \mathrm{~d} y \\
& +\int_{d(y, \partial \Omega)>\delta_{1}} \int_{\mathbb{R}^{Q}}(k(y+h, z)-k(y, z))^{2} \mathrm{~d} z \mathrm{~d} y \\
\leq & 4|\Omega|\|k\|_{L^{\infty}}^{2}\left|\left\{d(y, \partial \Omega) \leq \delta_{1}\right\}\right|+|\Omega|^{2} \frac{\varepsilon^{2}}{2|\Omega|^{2}} \\
\leq & \varepsilon^{2} .
\end{aligned}
$$

We conclude that $K$ is indeed compact on $L^{2}(\Omega)$.

\section{ACKNOWLEDGMENTS}

The authors wish to thank Benoit Perthame for the attention he paid to this work and Chris Cosner for pointing out the theory of line-sum-symmetric matrices that substantially improved the results.

\section{REFERENCES}

[1] Matthieu Alfaro and Jérôme Coville. Rapid traveling waves in the nonlocal Fisher equation connect two unstable states. Appl. Math. Lett., 25(12):2095-2099, 2012.

[2] Matthieu Alfaro, Jérôme Coville, and Gaël Raoul. Travelling waves in a nonlocal reactiondiffusion equation as a model for a population structured by a space variable and a phenotypic trait. Communications in Partial Differential Equations, 38(12):2126-2154, 2013.

[3] Matthieu Alfaro and Quentin Griette. Pulsating fronts for Fisher-KPP systems with mutations as models in evolutionary epidemiology. Nonlinear Anal. Real World Appl., 42:255-289, 2018.

[4] Anton Arnold, Laurent Desvillettes, and Céline Prévost. Existence of nontrivial steady states for populations structured with respect to space and a continuous trait. Commun. Pure Appl. Anal., 11(1):83-96, 2012.

[5] Guy Barles, Lawrence C. Evans, and Panagiotis E. Souganidis. Wavefront propagation for reaction-diffusion systems of PDE. Duke Math. J., 61(3):835-858, 1990.

[6] Henri Berestycki, Tianling Jin, and Luis Silvestre. Propagation in a non local reaction diffusion equation with spatial and genetic trait structure. Nonlinearity, 29(4):1434-1466, 2016. 
[7] Henri Berestycki, Grégoire Nadin, Benoît Perthame, and Lenya Ryzhik. The non-local FisherKPP equation: travelling waves and steady states. Nonlinearity, 22(12):2813, 2009.

[8] Emeric Bouin and Vincent Calvez. Travelling waves for the cane toads equation with bounded traits. Nonlinearity, 27(9):2233-2253, 2014.

[9] Emeric Bouin, Vincent Calvez, Nicolas Meunier, Sepideh Mirrahimi, Benoît Perthame, Gael Raoul, and Raphaël Voituriez. Invasion fronts with variable motility: phenotype selection, spatial sorting and wave acceleration. Comptes Rendus Mathematique, 350(15):761-766, 2012.

[10] Emeric Bouin, Christopher Henderson, and Lenya Ryzhik. The Bramson logarithmic delay in the cane toads equations. Quart. Appl. Math., 75(4):599-634, 2017.

[11] Emeric Bouin, Christopher Henderson, and Lenya Ryzhik. Super-linear spreading in local and non-local cane toads equations. J. Math. Pures Appl. (9), 108(5):724-750, 2017.

[12] Emeric Bouin, Christopher Henderson, and Lenya Ryzhik. The Bramson delay in the nonlocal Fisher-KPP equation. Annales de l'Institut Henri Poincaré C, Analyse non linéaire, 2019.

[13] Haim Brezis. Functional analysis, Sobolev spaces and partial differential equations. Universitext. Springer, New York, 2011.

[14] Vincent Calvez, Christopher Henderson, Sepideh Mirrahimi, Olga Turanova, and Thierry Dumont. Non-local competition slows down front acceleration during dispersal evolution. ArXiv e-prints, 2018.

[15] Robert Stephen Cantrell, Chris Cosner, and Yuan Lou. Evolutionary stability of ideal free dispersal strategies in patchy environments. J. Math. Biol., 65(5):943-965, 2012.

[16] Robert Stephen Cantrell, Chris Cosner, Yuan Lou, and Daniel Ryan. Evolutionary stability of ideal free dispersal strategies: a nonlocal dispersal model. Can. Appl. Math. Q., 20(1):15-38, 2012.

[17] Robert Stephen Cantrell, Chris Cosner, and Xiao Yu. Dynamics of populations with individual variation in dispersal on bounded domains. Journal of biological dynamics, 12(1):288-317, 2018.

[18] Robert Stephen Cantrell, Chris Cosner, and Xiao Yu. Populations with individual variation in dispersal in heterogeneous environments: Dynamics and competition with simply diffusing populations. Science China Mathematics, Jan 2020.

[19] Jack Dockery, Vivian Hutson, Konstantin Mischaikow, and Mark Pernarowski. The evolution of slow dispersal rates: a reaction diffusion model. J. Math. Biol., 37(1):61-83, 1998.

[20] Arnaud Ducrot, Thomas Giletti, and Hiroshi Matano. Spreading speeds for multidimensional reaction-diffusion systems of the prey-predator type. Calc. Var. Partial Differential Equations, 58(4):Art. 137, 34, 2019.

[21] B. Curtis Eaves, Alan J. Hoffman, Uriel G. Rothblum, and Hans Schneider. Line-sumsymmetric scalings of square nonnegative matrices. Number 25, pages 124-141. 1985. Mathematical programming, II.

[22] Grégory Faye and Matt Holzer. Modulated traveling fronts for a nonlocal Fisher-KPP equation: a dynamical systems approach. J. Differential Equations, 258(7):2257-2289, 2015.

[23] David Gilbarg and Neil S. Trudinger. Elliptic Partial Differential Equations of Second Order. Classics in Mathematics. Springer-Verlag, 2001.

[24] Léo Girardin. Non-cooperative Fisher-KPP systems: Asymptotic behavior of traveling waves. Mathematical Models and Methods in Applied Sciences, 28(06):1067-1104, 2018.

[25] Léo Girardin. Non-cooperative Fisher-KPP systems: traveling waves and long-time behavior. Nonlinearity, 31(1):108, 2018.

[26] Léo Girardin. Addendum to 'Non-cooperative Fisher-KPP systems: traveling waves and long-time behavior'. Nonlinearity, 32(1):168, 2019.

[27] Léo Girardin. Two components is too simple: an example of oscillatory Fisher-KPP system with three components. Proceedings of the Royal Society of Edinburgh: Section A Mathematics, pages 1-24, 2019.

[28] Quentin Griette. Singular measure traveling waves in an epidemiological model with continuous phenotypes. Trans. Amer. Math. Soc., 371(6):4411-4458, 2019.

[29] Quentin Griette and Gaël Raoul. Existence and qualitative properties of travelling waves for an epidemiological model with mutations. J. Differential Equations, 260(10):7115-7151, 2016.

[30] Quentin Griette, Gaël Raoul, and Sylvain Gandon. Virulence evolution at the front line of spreading epidemics. Evolution, 69(11):2810-2819, 2015.

[31] Aled Morris, Luca Börger, and Elaine C. M. Crooks. Individual variability in dispersal and invasion speed. Mathematics, 7(9), 2019.

[32] Olga Turanova. On a model of a population with variable motility. Math. Models Methods Appl. Sci., 25(10):1961-2014, 2015. 
[33] Hans F. Weinberger. Invariant sets for weakly coupled parabolic and elliptic systems. Rend. Mat. (6), 8:295-310, 1975. Collection of articles dedicated to Mauro Picone on the occasion of his ninetieth birthday. 